\title{
39. On the Termination Effects in the Fourier Analysis of a Crystal
}

\author{
By Kenji Dor \\ Mineralogical Institute, University of Tokyo \\ (Comm. by T. Iто, M.J.A., March 12, 1954)
}

The Fourier synthesis of a crystal is unavoidably accompanied by the so-called termination effects, which, giving rise to a number of false peaks in the resulting diagram, present difficulties in locating the precise positions of atoms. The writer will put forward in the following a convenient procedure that, without assumption as to the chemistry of constituent atoms, might eliminate such ghosts and lead to a correct reading of the Fourier map of electron density. This method would provide, by enabling us in the end to estimate the amount of errors due to the termination of Fourier series, a useful means for objectively delineating the most probable electron configuration of atoms and the nature of bonds in a crystal.

In a one-dimensional crystal with period $a$, we have for the electron density $\rho(x)$, when free from the termination effects:

$$
\rho(x)=\frac{1}{a} \sum_{h=-\infty}^{+\infty} F_{h} e^{-\frac{2 \pi i}{\lambda} \cdot \frac{h x}{a}} .
$$

This reduces, when the series is cut off at $H^{\prime}$ th term, to

$$
\rho_{H}(x)=\frac{1}{a} \sum_{h=-H}^{+H} F_{h} e^{-\frac{2 \pi i}{\lambda} \cdot \frac{h x}{a}} .
$$

Pepinsky ${ }^{1)}$ pointed out the significance of Dirichlet's kernel in the problem of Fourier summation and obtained:

$$
\begin{aligned}
\rho_{H}(x) & =\frac{2}{a} \int_{-\alpha / 2}^{+a / 2} \rho(x+u) \frac{\sin 2 \pi\left(H+\frac{1}{2}\right) \frac{u}{a}}{2 \sin 2 \pi \frac{u}{2 a}} d u \\
& =\frac{2}{a} \int_{-a / 2}^{+a / 2} \rho(x+u) D_{H}(u) d u,
\end{aligned}
$$

where

$$
D_{H}(x)=\frac{\sin 2 \pi\left(H+\frac{1}{2}\right) \frac{x}{a}}{2 \sin 2 \pi \frac{x}{2 a}} .
$$

The relation shows that $\rho_{H}(x)$ arises from convolution of $D_{H}(x)$ with $\rho(x)$ and we can reduce, if we know the properties of $D_{H}(x), \rho_{H}(x)$ to $\rho(x)$ that represents the electron distribution without distortion 
due to the termination effects. $D_{H}(x)$ is an even function and has, in addition to the principal maximum at the origin, $(H-1)$ subsidiary maxima, the value of which diminishes as $x$ increases from 0 to $1 / 2 a$.

For concreteness, let us assume a one-dimensional 'oxygen crystal' 'in which $O$-atoms are placed at the origin and other points

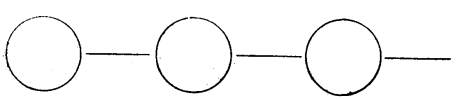

$\leftarrow a=5.28 \AA \rightarrow$

Fig. 1. Oxygen atoms placed linearly at points each $5.28 \AA$ apart

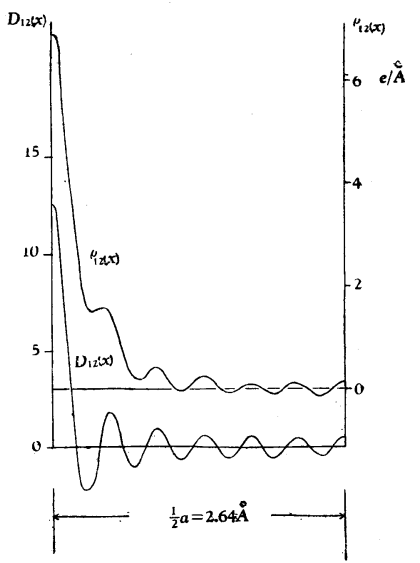

Fig. 2. $\rho_{12}(x)$ and $D_{12}(x)$ curves corresponding to Fig. 1

their principal and subsidiary peaks at the same $x$ 's. This is because the maxima in $\rho_{H}(x)$ may be thought of as taking place as the results of a Patterson interaction between each of $D_{H}(x)$ maxima and the maximum in $\rho(x)$ due to the oxygen atom at the origin. Therefore, we can, starting from $D_{H}(x)$ curve, readily point out in $\rho_{H}(x)$ curve the fortuitous peaks that should be discarded as simply due to convolution.

In order to apply the result obtained in the foregoing discussion each $5.28 \AA$ apart (Fig. 1). We assume that oxygen atoms have a Hartree distribution of electrons and calculate $F_{H}$-values with $h=0$ to $h=12$. The one-dimensional synthesis using these $F$-values: $\rho_{12}(x)=\frac{1}{a} \sum_{h=-12}^{+12} F_{h} e^{-\frac{2 \pi i}{\lambda} \cdot \frac{h x}{a}}$ is represented in Fig. 2, together with $D_{12}(x)$ curve. These two curves have
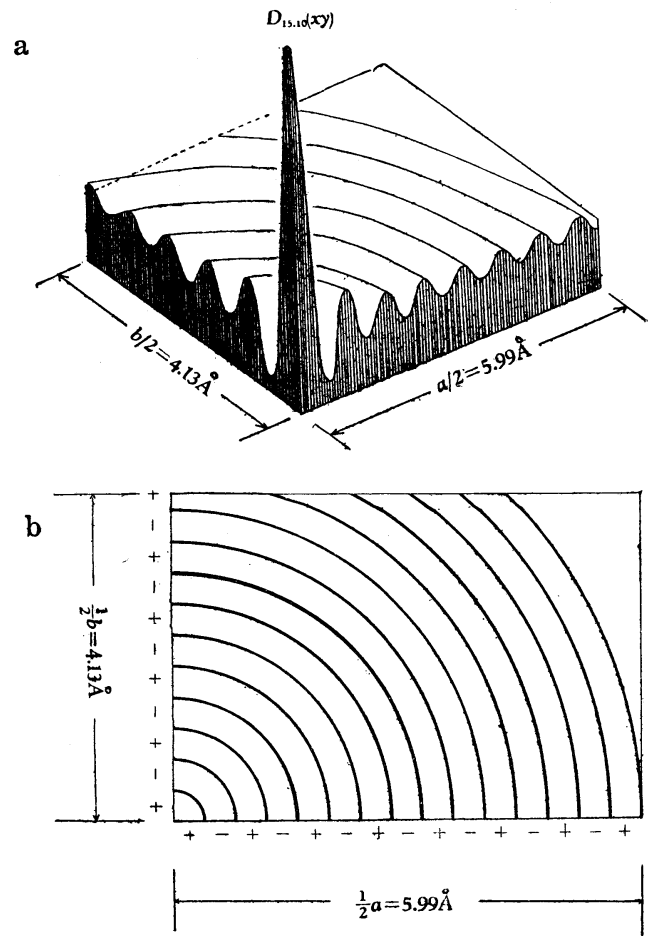

a: In perspective $b$ : In projection

Fig. 3. A two-dimensional $D$-diagram drawn using the numericals given by Lundgren for $\mathrm{CeOSO}_{4} \cdot \mathrm{H}_{2} \mathrm{O}$ 
to the two-dimensional Fourier projection, we revolve, for practical purposes, the $D$-diagram about the ordinate, obtaining a two-dimen-

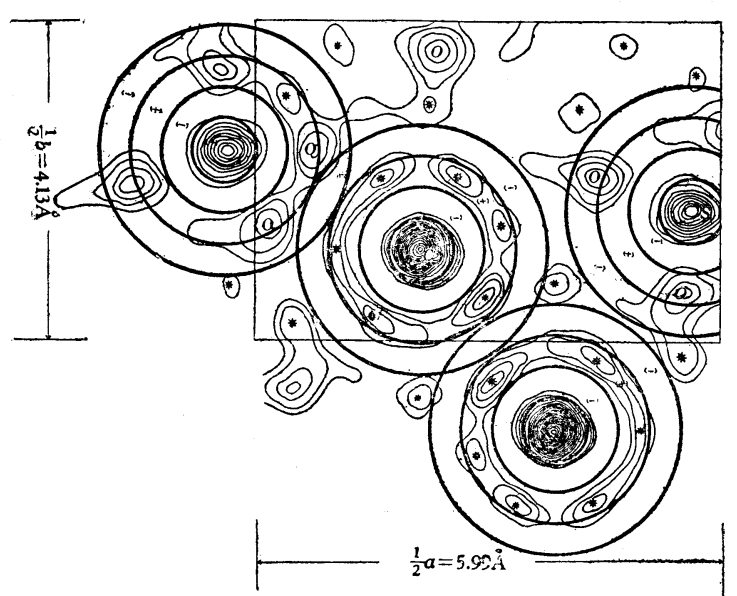

Fig. 4. Fourier (001) projection of $\mathrm{CeOSO}_{4} \cdot \mathrm{H}_{2} \mathrm{O}$ (after Lundgren) superimposed by Dirichlet's kernel $D_{15,10}(x, y)$. The ghosts to be discarded are marked by asterisks. sional $D$-diagram consisting of concentric zones of crests and troughs as shown in Fig. 3.* We have to place such a diagram upon the Fourier diagram at every point of the unit plane where a heavy atom is seen projected. Fig. 4 shows the Fourier map of $\mathrm{CeOSO}_{4} \cdot \mathrm{H}_{2} \mathrm{O}$ published by Lundgren") superimposed by the relevant $D$-diagrams. We can now easily discriminate the real from spurious atomic positions in the projection, the latter lying for the most part in the positive zones of the $D$-diagrams overlapped.

The writer wishes to express his sincere gratitude to Professor T. Ito for suggestion and encouragement.

\section{References}

1) R. Pepinsky: Computing Methods and the Phase Problem in X-ray Crystal Analysis, p. 319. Pennsylvania State College (1952).

2) G. Lundgren: Arkiv f. Kemi, 6, 59 (1953).

*) We assume that the reciprocal space experimentally observable is spherical. 